\title{
Study of Long Range van der Waals Interactions with Liquid-Phase STEM
}

\author{
Elisa Cepeda-Pérez ${ }^{1}$, Andreas Verch ${ }^{1}$ and Niels de Jonge ${ }^{1,2}$ \\ 1. INM - Leibniz Institute for New Materials, Saarbrücken, Germany. \\ 2. Department of Physics, University of Saarland, Saarbrücken, Germany.
}

The interplay of short range- $(<10 \mathrm{~nm})$ and long-range forces influence the dynamics of colloidal systems in liquid. Colloidal nanoparticles are subject to two major types of attractive forces, van der Waals force and dipolar electrostatic interactions, and to electrostatic repulsive force [1]. Electrostatic interactions are well understood, and are typically the strongest force between nanoparticles at nanometer distance. But ions in the liquid shield electrostatic interactions from propagating into the long range. On the contrary, the van der Waals forces are not shielded. Even when van der Waals forces are not as strong as electrostatic or H-bonding forces, they play a key role in the stability of colloidal suspensions [2]. Certain aspects of the interactions between nanoparticles are still not well understood [3]. For example, van der Waals interactions are typically only considered for short range $(<10 \mathrm{~nm})$ interactions. However, recent reports demonstrate that van der Waals interactions ranging out to tens of nanometers and are significant, and contribute to the assembly between colloidal nanoparticles [2].

We utilized liquid-phase scanning transmission electron microscopy (STEM) to observe the motion trajectories of individual gold nanoparticles (AuNPs) in solution during self-assembly- and other dynamic processes. The experimental setup consisted of a liquid cell formed by two silicon microchips, each containing a $50 \mathrm{~nm}$-thick electron transparent silicon nitride ( $\mathrm{SiN}$ ) window. We followed the previously reported procedure for liquid-phase STEM sample preparation [4]. To ensure a defined distance between the chips, one of the silicon microchips included a spacer of $0.5 \mu \mathrm{m}$ height. A solution containing $20 \mathrm{~nm}$-diameter AuNPs was placed between the two silicon chips. Since the dynamical movements of nanoparticles within the liquid cell are orders of magnitude slower than what would be expected based on Brownian motion under the applied experimental conditions [4], it is possible to observe the clustering processes within the temporal capabilities of STEM. We acquired a time lapse STEM image series, and analyzed the trajectories and distances between the AuNPs as function of time. Our experimental data show how the dynamical movements of the AuNPs were driven by both repulsive- and attractive forces between the particles. Attractive van der Waals interactions were dominant when the distance between the surfaces of the AuNPs was in the range of 20-100 nm. Unlike the interactions caused by collisions due to Brownian movement, the AuNPs followed specific trajectories that minimized the distance between them.

In summary, we observed and analyzed the nanoscale interactions between spherical AuNPs by liquidphase STEM. We found that the dynamical behavior of the AuNPs at the solid-liquid interface involved long-range attractive interactions that led to the formation of clustered structures. This finding reveals the importance of the attractive forces between colloidal nanoparticles during self-assembly processes in under conditions of reduced Brownian motion [5].

References:

[1] JN Israelachvili in "Intermolecular and surface forces", (Academic Press, Waltham, 2011).

[2] Y Lalatonne, J Richardi, and M Pileni, Nat Mater 3 (2004), p. 121. 
[3] GA Rance et al, ACS Nano 4 (2010), p. 4920.

[4] A Verch, M Pfaff, and N de Jonge, Langmuir 31 (2015), p. 6956.

[5] We thank Eduard Arzt for his support through INM.

A

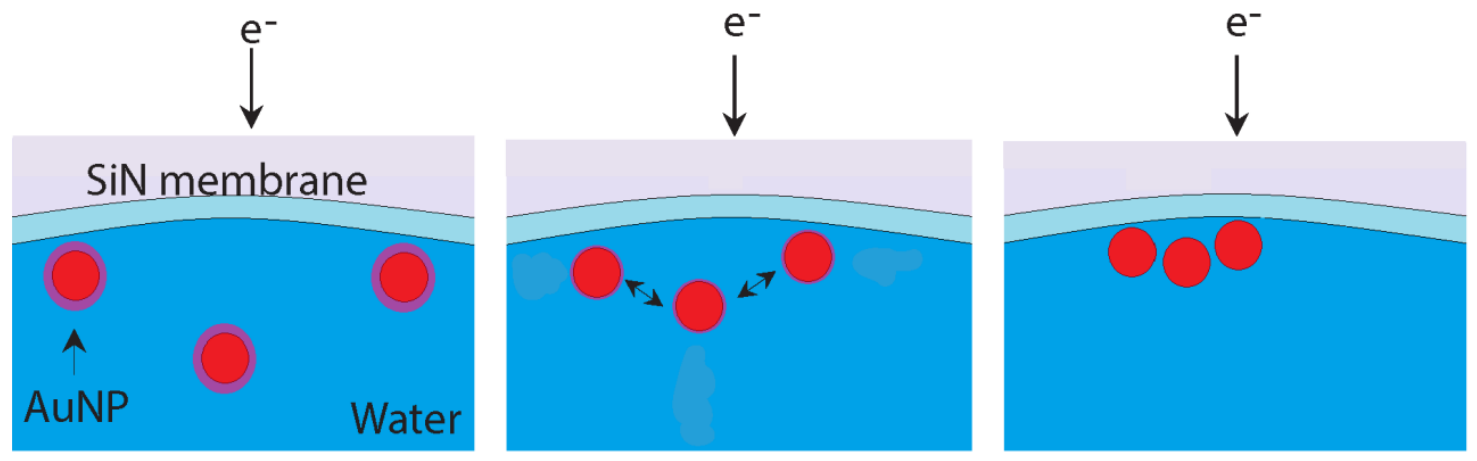

B

$2.9 \mathrm{~s}$

$13 \mathrm{~s}$

$34.8 \mathrm{~s}$
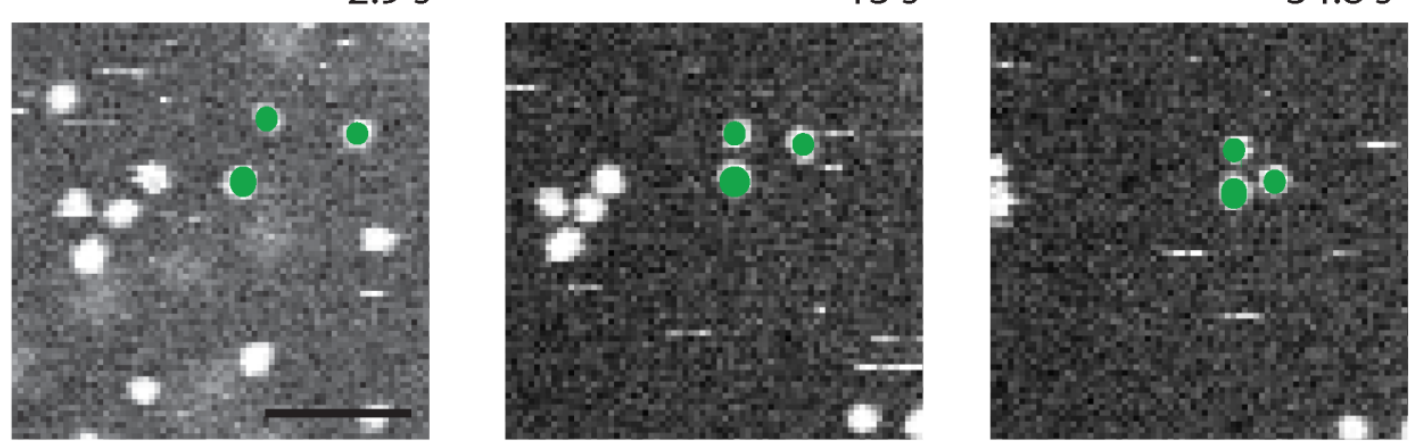

Figure 1. Observing long-range van der Waals interactions between AuNPs using liquid-phase STEM. (A) Schematic representation of the interaction of AuNPs in liquid upon electron beam irradiation. Negatively charged AuNPs are initially dispersed and located in the in the vicinity of the SiN membrane. The interaction of the electron beam with the water alters the chemistry of the system and modifies the repulsive forces that kept the AuNPs dispersed. The result of electron beam irradiation is the movement of AuNPs towards each other. (B) Stills from an annular dark-field STEM time-lapse series at different time points of $20 \mathrm{~nm}$-diameter AuNPs in a solution containing 10\% phosphate buffered saline (PBS). The electron dose rate was $3.3 \mathrm{e}^{-} / \mathrm{s}^{2}$. Scale bar $100 \mathrm{~nm}$. 